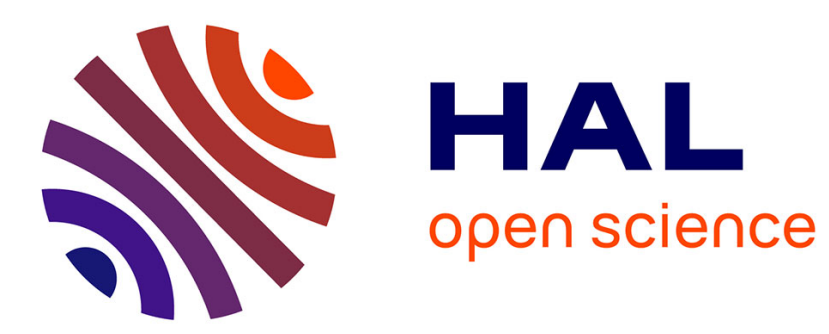

\title{
General Uncertainty in Portfolio Selection: A Case-Based Decision Approach
}

\author{
Vasyl Golosnoy, Yarema Okhrin
}

\section{To cite this version:}

Vasyl Golosnoy, Yarema Okhrin. General Uncertainty in Portfolio Selection: A Case-Based Decision Approach. Journal of Economic Behavior and Organization, 2008, 67 (3-4), pp.718. 10.1016/j.jebo.2007.08.004 . hal-00614677

\section{HAL Id: hal-00614677 \\ https://hal.science/hal-00614677}

Submitted on 15 Aug 2011

HAL is a multi-disciplinary open access archive for the deposit and dissemination of scientific research documents, whether they are published or not. The documents may come from teaching and research institutions in France or abroad, or from public or private research centers.
L'archive ouverte pluridisciplinaire HAL, est destinée au dépôt et à la diffusion de documents scientifiques de niveau recherche, publiés ou non, émanant des établissements d'enseignement et de recherche français ou étrangers, des laboratoires publics ou privés. 


\section{Accepted Manuscript}

Title: General Uncertainty in Portfolio Selection: A

Case-Based Decision Approach

Authors: Vasyl Golosnoy, Yarema Okhrin

PII: $\quad$ S0167-2681(07)00170-9

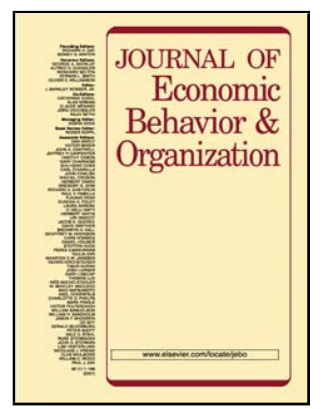

DOI: doi:10.1016/j.jebo.2007.08.004

Reference: $\quad$ JEBO 2142

To appear in: Journal of Economic Behavior \& Organization

Received date: $\quad$ 15-3-2006

Revised date: 21-8-2007

Accepted date: $\quad 23-8-2007$

Please cite this article as: Golosnoy, V., Okhrin, Y., General Uncertainty in Portfolio Selection: A Case-Based Decision Approach, Journal of Economic Behavior and Organization (2007), doi:10.1016/j.jebo.2007.08.004

This is a PDF file of an unedited manuscript that has been accepted for publication. As a service to our customers we are providing this early version of the manuscript. The manuscript will undergo copyediting, typesetting, and review of the resulting proof before it is published in its final form. Please note that during the production process errors may be discovered which could affect the content, and all legal disclaimers that apply to the journal pertain. 


\section{Introduction}

The economic literature differentiates between several kinds of uncertainty (cf. Dequech 2006). According to Knight (1921), uncertainty characterizes situations where the states of nature are known but their probabilities are not. The multi-prior maximin expected utility (EU) approach of Gilboa and Schmeidler (hereafter 'GS') (1989) allows us to model this type of uncertainty. However, such an approach is inappropriate for decision problems with general uncertainty where neither states of nature nor their probabilities are known. GS (1995, 2001a) develop the case-based decision theory (CBDT) for decision-making in situations with general uncertainty. The CBDT is applied to economic problems, among others, by GS (1997b, 2001b), Blonski (1999), and Jahnke et al. (2005).

The portfolio investor encounters general uncertainty because it is often hard to imagine all states of nature relevant for the asset allocation problem. In particular, general uncertainty can be seen as uncertainty whether the pre-selected asset allocation model is correct or/and the underlying assumptions are valid. To our knowledge there is no literature dealing with this type of uncertainty in portfolio context. This paper aims is to fill this gap and to assess general uncertainty in portfolio selection with the tools of the case-based reasoning. We adopt the CBDT for this purpose and investigate which case-based strategies lead to portfolio performance improvement.

We quantify general uncertainty as a degree of the investor's distrust in the possibility of achieving positive utility effects from investing according to the pre-selected formal model for asset allocation. The time-varying model belief degree is considered as a proxy for general uncertainty. The dynamic choice of the model belief degree is implemented using a case-based approach in a two stage procedure. In the first stage the investor applies a conventional portfolio rule, while in the second stage he uses case-based reasoning for determining his degree of model belief. The CBDT investor makes decisions solely using his experience, past performance and similarity of past situations to the present. The similarity function quantifies the distance between two situations; its form depends on the value of the indifference parameter. The decision to choose should be close to decisions in situations with favorable and far from those in situations with unfavorable past outcomes. The aspiration level distinguishes between favorable and unfavorable outcomes. It is adjusted using incoming information.

The case-based approach amends the EU reasoning in situations without information about possible states of nature (GS 2001a, p. 27ff). The linkage between the CBDT and EU rules is shown by Matsui (2000). The CBDT is grounded on analogical thinking where current preferences depend on past experience. It implies bounded rationality in the sense of March and Simon (1958). Deviations from rationality influence the formation of general equilibrium due to limits to arbitrage. Behavioral phenomena arise due to biases in people's belief formation and/or due to preferences concerning decision-making based on given beliefs (cf. Barberis and Thaler 2003). Alternatively to the EU approach, the prospect theory of Kahneman and Tversky (1979) and its successors provides a successful description of economic agent preferences. On the contrary, the CBDT is not an alternative, but an amendment to the EU and prospect theories. Our case-based investor may exhibit the representativeness bias (too much weight on recent patterns in the data), conservatism (too much weight on prior beliefs), anchoring (too much importance on the starting value) or availability biases. However, we see the focus of this paper not on explaining behaviorial effects but on exploring which patterns of case-based decisions could be of interest for the portfolio investor.

The agent considered in the paper is an uninformed price-taker, maximizing his EU for a given risk 
aversion coefficient. He is not a representative investor, but merely a person willing to account for general uncertainty by means of the case-based approach. The indifference and memory parameter values determine his case-based strategy. The empirical study explores which case-based strategies bring the best investment results. Pure EU maximization strategies and simple portfolio rules as well as modified EU approaches are used with and without case-based amendment. The CBDT investor with a small risk aversion achieves the best EU results for good memory and high indifference degree. Such an investor may exhibit anchoring and conservatism biases. His aspiration level and model belief degree would be gradually adjusted to new information. This corresponds to the fundamental trading strategy oriented on predictability of the future portfolio performance by the past one. On the contrary, the investor with a large risk aversion mostly benefits when his CBDT strategy is characterized by poor memory and a low indifference degree. This implies quick adjustments of the aspiration level and model belief degree. Such an investor may exhibit availability and representativeness biases. This is a variation of the active trading strategy, implying improvement of arbitrage on financial markets.

The empirical evidence provides insights about the link between case-based reasoning and market efficiency. Active trading should be profitable on markets with a high efficiency degree, while fundamental trading is more appropriate for non-efficient markets, characterized by a higher degree of predictability. Investigating the portfolio of Dow Jones Industrial stocks, we find that benefits of the fundamental strategy reduce over time, while the positive effects of following the active trading case-based strategy increase. This supports the hypothesis of increasing US market efficiency. The active case-based trader quickly reacts to newcoming information. Such investment strategy improves market efficiency and may survive in financial markets in the long run. These findings shed light on the consequences of applying CBDT to model uncertainty (MU) assessment in portfolio selection.

The rest of the paper is organized as follows. Section 2 discusses the issues of risk and uncertainty in portfolio selection. Our case-based methodology to assessment of general uncertainty in portfolio problems is introduced in Section 3. Section 4 provides the empirical study, while Section 5 concludes the paper. The technical details are provided in the Appendix and in the supplement available on the JEBO website.

\section{Risk and Uncertainty in Portfolio Selection}

\subsection{Asset Allocation under Uncertainty}

The conventional wealth allocation approach suggests choosing portfolio weights by maximizing the investor's EU. In the Markowitz (1952) framework, the EU is maximized in the mean-variance procedure by finding the optimal trade-off between the expected portfolio return and risk associated with future investment outcomes. The unknown model parameters cause estimation risk, its importance recognized since Klein and Bawa (1976). Estimation errors, especially those in expected returns, seriously hamper portfolio performance (Best and Grauer, 1991). Bayesian (Jorion 1986, Polson and Tew 2000, Kumar 2006) or frequentistic (Ledoit and Wolf 2003, ter Horst et al. 2006, Kan and Zhou 2006, Golosnoy and Okhrin 2007) methods reduce of the estimation risk in portfolio selection. The portfolio rule should be adjusted using one or a combination of the aforementioned methods. 
However, the EU-based portfolio strategies often perform unsatisfactorily even after adjusting for estimation risk (Michaud, 1998). They may be unable to beat simple benchmark approaches in terms of the out-of-sample EU. Constraining portfolio weights (Frost and Savarino, 1988, Grauer and Shen, 2000, Jagannathan and Ma, 2003) or different modifications to equal weight portfolio strategies (Black and Litterman, 1992, DeMiguel et al., 2008) frequently outperform Markowitz-based rules. The non-satisfactory performance points at possible misspecification of the pre-selected models. The investor remains unaware of the true model; thus accounting for MU can mitigate the negative effects of false model assumptions.

Economic literature differentiates uncertainty types depending on the amount of information about states of nature and their probabilities. According to Knight, the issue of risk refers to situations where the states of nature as well as their probabilities are given, while uncertainty refers to situations where the states of nature are given but the probabilities are unknown. Moreover, there exist situations in which the decision-maker faces general uncertainty or structural ignorance (cf. GS 2001a), where even states of nature are neither known nor can be easily constructed. The EU framework is appropriate for decision making under risk. GS (1989) propose the maximin EU approach for situations with unknown probabilities and known states of nature. However, the EU rule is inappropriate for situations with unknown probabilities and states of nature. GS (1995, 2001a) develop the CBDT for decision-making in such situations. The case-based economic agent makes decisions using his experience and the similarity of encountered situations to the current one. Next we discuss the assessment of Knightian and general uncertainty in portfolio selection.

\subsection{Assessment of Knightian Uncertainty}

Decision making under Knightian uncertainty is grounded on the papers of GS (1989) and Chamberlain (2000). Their probabilistic maximin approach implies the choice of the EUs over the set of alternative prior distributions. The application of maximin to MU is based on choosing the most favorable variant from the set of least favorable alternatives. The maximin-based multiprior approaches are widely used for uncertainty modeling in economic applications (cf. Hansen and Sargent 2001, Kogan and Wang 2003, Aiolfi and Favero 2005).

An elegant way to quantify the degree of Knightian uncertainty in portfolio context in a Bayesian framework is developed by Pastor (2000) and Pastor and Stambaugh (2000). The prior variance of the intercept in asset-pricing factor models serves as a proxy for the uncertainty degree. The size of the variance determines the trade-off between the influence of the prior density and of the likelihood function on the posterior density. In the case of low uncertainty, the prior plays a more important role; otherwise the investor gives more weight to the likelihood (i.e. to the data). Avramov (2002) assesses MU by averaging alternative forecasting models, extending the approach of Pastor. Tu and Zhou (2004) additionally consider the uncertain data-generating process (DGP) by means of Bayesian updating of prior beliefs about the true DGP.

The quantification of Knightian uncertainty in portfolio selection is combined with decision making in a multiprior framework by Wang (2005) and Garlappi et al. (2007). Wang extends the approach of Pastor by considering an investor with a maximin portfolio problem,

$$
\max _{\mathbf{w}} \min _{\omega} E U(\mathbf{w}, \omega)
$$


where the $\mathrm{EU}$ is maximized with respect to the portfolio weights $\mathbf{w}$ and minimized with respect to the MU degree $\omega$. The approach of Wang (2005) is Bayesian; the prior variance of $\omega$ determines the degree of uncertainty, which could be seen as a degree of trust in the factor model. Garlappi et al. propose a frequentistic multiprior approach to MU. They impose additional constraints on model parameters, allowing a wide range of possible alternative distributions for the uncertain values. The size of the constraints influences asset allocation and represents the degree of Knightian uncertainty. This approach is based on selecting from a set of different models; unfortunately it is limited by a relatively small number of alternatives.

However, it remains unclear why MU should be Knightian (i.e. limited by any clearly restricted set of alternatives). In real economic situations there is uncertainty about the underlying distribution of asset returns or whether this distribution will be unchanged in the future. The investor may be uncertain whether his investment opportunities are representative enough as well as about systematic risk of his investments. Hence, there are very different uncertainty types, so it may be difficult to imagine all states of nature, not even considering assigning utility outcomes to each possible state. The EU reasoning is inappropriate for this type of modeling, where analogy-based methods should be used (GS 2001a, p. 45ff).

\subsection{Assessment of General Uncertainty}

The portfolio investor unable to imagine possible states of nature faces general uncertainty. GS (2001a) suggest using the tools of the CBDT for making decisions in these situations. The case-based reasoning amends the EU rule and is grounded not on probabilistic but on psychological aspects of decision-making. Next we propose a way of adjusting conventional portfolio rules by introducing a degree of general uncertainty.

We relate general uncertainty to the strength of an investor's belief in the chosen model. Uncertainty is quantified as a degree of disbelief in the possibility of achieving some EU gains by investing in risky assets compared to the risk-free alternative. The degree of model belief at time point $t$ is denoted by $\theta_{t} \in[0 ; 1]$ where $\theta_{t}=0$ stands for complete disbelief while $\theta_{t}=1$ indicates complete belief. The interval is restricted from zero to unity in order to escape model "overconfidence" $\theta_{t}>1$ and "negative belief" $\theta_{t}<0$. These issues remain beyond the scope of the paper, but may occur in practical situations. Accordingly, the degree of disbelief is quantified by $1-\theta_{t}$.

The portfolio strategy with general uncertainty is described as follows. The optimal portfolio weights $\hat{\mathbf{w}}_{t}$ at time point $t$ are estimated using one of the portfolio selection models. We suggest using the model belief degree for the linear weighting of wealth proportions chosen with a non-CBDT rule. The optimal weights of risky assets adjusted to the model belief degree are given by

$$
\hat{\mathbf{v}}_{t}=\theta_{t} \hat{\mathbf{w}}_{t}
$$

This way of modeling originates from the idea of adjusting portfolio composition by moving along the capital market line (CML). If the degree of model belief $\theta_{t}$ decreases, the investor moves on the CML towards the risk-free rate. This is equivalent to choosing a larger coefficient of risk aversion, similar to the idea of ter Horst et al. 


\section{Case-Based Approach to General Uncertainty}

Now we introduce the CBDT approach for determining the model belief degree $\theta_{t}$. First we describe the essence of the case-based approach, then we adopt the CBDT for our purposes. Finally, we discuss the role of case-based reasoning in portfolio selection.

\subsection{The Essence of Case-Based Approach}

The CBDT grounds decision making on previous experience and on the similarity of previous situations to the current one. The economic agent chooses current action by maximizing utilities of previous outcomes weighted by a similarity function. The similarity function provides the distance between the current and previously encountered problem-act pairs. GS (1997a) introduce similarity of acts for situations where the same problem should be solved many times and there is a large number of different acts to choose from.

To formalize the approach let $\Theta$ denote the discrete set of possible/experienced acts. The CBDT selects the decision $\theta_{t}^{*} \in \Theta$ at the time $t$ maximizing the objective function $Q\left(\theta_{t}\right)$

$$
\theta_{t}^{*}=\arg \max _{\theta_{t} \in \Theta} Q\left(\theta_{t}\right) \quad \text { with } \quad Q\left(\theta_{t}\right)=\sum_{\theta \in \Theta}\left(U(\theta)-H_{t}\right) s\left(\theta, \theta_{t}\right)
$$

The objective function is a weighted sum of the excess utilities, calculated as the difference between realized utilities of possible outcomes $\theta \in \Theta$ and the aspiration level $H_{t}$, measuring the decision maker's satisfaction. The aspiration $H_{t}$ differentiates between favorable and unfavorable outcomes and is updated with incoming information. The similarity function $s\left(\theta, \theta_{t}\right)$ penalizes the distance between decision $\theta_{t}^{*}$ and possible outcomes $\theta \in \Theta$. The current decision (act) $\theta_{t}^{*}$ should be chosen "close" to decisions in situations with favorable and "far" from decisions in situations with unfavorable outcomes. The distance depends on the indifference parameter; low indifference indicates large distance even between similar situations. The CBDT decision maker assumes that acts favorable for a current problem in the past will also be favorable in the future. Thus the chosen $\theta_{t}^{*}$ can be seen as a CBDT forecast for the optimal model belief degree in the next period.

The implementation of case-based procedure depends primarily on the choice of the similarity function and the way the aspiration level is updated. Extensive discussion of these issues for various economic situations is provided by GS (2001a).

\subsection{Selecting Degree of Uncertainty}

The act similarity, proposed by GS (1997a), is appropriate for our problem because it remains the same for all periods. The belief degree $\theta_{t}$ specifies the act and reflects investor's uncertainty. We consider a continuous set of possible acts and determine the optimal degree of model belief $\theta_{t}^{*} \in[0 ; 1]$ by maximizing the case-based objective function $Q\left(\theta_{t}\right)$ :

$$
\theta_{t}^{*}=\underset{\theta_{t} \in[0,1]}{\arg \max } Q\left(\theta_{t}\right), \quad \text { with } \quad Q\left(\theta_{t}\right)=\int_{0}^{1} \Delta U_{t}(\theta) s\left(\theta_{t}, \theta\right) d \theta,
$$


where $s(\cdot)$ is a similarity function for acts, $\Delta U_{t}(\theta)=U_{t}(\theta)-H_{t}$ is a hypothetical excess utility for a fixed $\theta$, and $H_{t}$ denotes the aspiration level. In the classical CBDT (e.g. by visiting different restaurants), a person chooses just one and remains unaware of realizations of other alternatives. Our investor can easily determine the results of all hypothetically possible choices of $\theta$ at any point of time. Thus we calculate the hypothetical excess utilities $\Delta U_{t}(\theta)$ for each choice of $\theta \in[0 ; 1]$ and then select the optimal $\theta_{t}^{*}$ by maximizing $Q\left(\theta_{t}\right)$. Gilboa et al. (2002) axiomatize hypothetical reasoning.

The hypothetical CBDT returns $r_{p, i}(\theta)$ at time $i=1, \ldots, t$ for any $\theta$ are given by

$$
r_{p, i}(\theta)=\theta \hat{\mathbf{w}}_{i-1}^{\prime} \mathbf{r}_{i}+\left(1-\theta \hat{\mathbf{w}}_{i-1}^{\prime} \mathbf{1}\right) r_{f}=\theta\left(r_{p, i}-r_{f}\right)+r_{f},
$$

where $\hat{\mathbf{w}}_{i-1}$ denotes the portfolio composition at time $i-1$ and $r_{p, i}$ denotes the realized portfolio return from portfolio rules without general uncertainty. Thus for each $\theta$ we get a time series of portfolio returns $\left\{r_{p, i}(\theta)\right\}$ of length $t$. Then we calculate the hypothetical utility function by

$$
U(\theta)=\theta E\left(r_{p}\right)+(1-\theta) r_{f}-\frac{\gamma \theta^{2}}{2} V\left(r_{p}\right)
$$

The expectation $E\left(r_{p}\right)$ and variance $V\left(r_{p}\right)$ are estimated using the whole history of realized $\left\{r_{p, i}\right\}$. Here we neglect the variability in $r_{f}$ because it is tiny compared to those of risky assets.

The aspiration level is a "happiness point" of the investor. The acts outperforming the aspiration level are perceived as favorable, while underperforming acts are regarded as unfavorable. We do not consider exogenous (Oechssler, 2002) or uncertain aspirations (MacLeod and Pingle, 2005), but use endogenous aspirations depending on individual payoff history only. Our endogenous aspiration level is updated with outcomes of the investor's acts. Because the portfolio problem stays the same, the aspiration updating corresponds to the investor's learning procedure, similar to game theory literature (Boergers and Sarin, 2000). We utilize the proposition of GS (2001a, p. 155ff) to update aspirations according to the exponential rule,

$$
H_{t}=(1-\lambda) H_{t-1}+\lambda u_{t},
$$

with a smoothing $\lambda \in[0 ; 1]$ denoting the memory strength. The exponential rule is widely used for adjustments of the aspiration level for innovations (cf. Hanaki et al. 2005). GS (2001a, p. 159ff) argue that the CBDT can also be used with some other aspiration updating rules. The aspiration innovations are defined by $u_{t}=r_{p, t}\left(\theta_{t-1}^{*}\right)-\gamma r_{p, t}^{2}\left(\theta_{t-1}^{*}\right) / 2$. The aspiration level can be written as

$$
H_{t}=H_{0}+\lambda \sum_{i=1}^{t}(1-\lambda)^{t-i} r_{p, i}\left(\theta_{i-1}^{*}\right)-\frac{\gamma}{2} \lambda \sum_{i=1}^{t}(1-\lambda)^{t-i} r_{p, i}^{2}\left(\theta_{i-1}^{*}\right)
$$

where $H_{0}$ denotes the initial level. Thus it is calculated by exponential smoothing of the realized $r_{p, t}\left(\theta_{t-1}^{*}\right)$ and squared $r_{p, t}^{2}\left(\theta_{t-1}^{*}\right)$ CBDT returns.

The choice of the similarity function is of great importance for the case-based reasoning. We consider the modulus similarity function:

$$
s\left(\theta_{i}, \theta_{j}\right)=1-\left|\theta_{i}-\theta_{j}\right|^{\kappa} .
$$


Other similarity functions may also be of interest (Billot et al., 2005). The indifference parameter $\kappa \in(0,+\infty)$ determines the form of the similarity function. Small values of $\kappa$ lead to a large distance even between very similar acts. The modulus similarity function is linear for $\kappa=1$, concave for $\kappa>1$ and convex for $\kappa<1$.

To simplify the problem (3), we provide an explicit expression for the objective function $Q\left(\theta_{t}\right)$ for the given form of hypothetical utility (5), aspiration adjustments (6) and similarity function (8). Proposition 1 is obtained by integrating out $\theta$ from $Q\left(\theta_{t}\right)$.

Proposition 1. For the modulus similarity function $s\left(\theta_{i}, \theta_{j}\right)=1-\left|\theta_{i}-\theta_{j}\right|^{\kappa}$ with $\kappa \in(0,+\infty)$, it holds that the case-based objective function $Q\left(\theta_{t}\right)$ from (3) is given by

$$
\begin{aligned}
& Q\left(\theta_{t}\right)=\left(r_{f}-H_{t}\right) \times c_{1}+\left(E\left(r_{p}\right)-r_{f}\right) \times c_{2}-\frac{\gamma}{2} V\left(r_{p}\right) \times c_{3}, \\
& \text { with } \quad c_{1}=1-\frac{\theta_{t}^{\kappa+1}+\left(1-\theta_{t}\right)^{\kappa+1}}{\kappa+1}, \quad c_{2}=\frac{1}{2}-\frac{\theta_{t}^{\kappa+2}+\left(1-\theta_{t}\right)^{\kappa+1}\left(1+\kappa+\theta_{t}\right)}{(\kappa+1)(\kappa+2)}, \quad \text { and } \\
& c_{3}=\frac{1}{3}-\frac{2 \theta_{t}^{\kappa+3}+\left(1-\theta_{t}\right)^{\kappa+1}\left[(1+\kappa)\left(1-\theta_{t}\right)+\left(1+\kappa+\theta_{t}\right)\left(1+\kappa+2 \theta_{t}\right)\right]}{(\kappa+1)(\kappa+2)(\kappa+3)} .
\end{aligned}
$$

The influence of the similarity function $s(\cdot)$ on the objective $Q\left(\theta_{t}\right)$ is complex. The investor perceives the similarity of different portfolio choices based on the indifference parameter $\kappa$, which ascertains how strongly the investor distinguishes between different $\theta$ s. Moreover, the $\kappa$ value determines the inclination of the investor to the sub-optimal choices. A value of $\kappa$ close to zero would give the unit weight to the largest utility and zero weights for the utilities with other $\theta$ s. In this case the optimal $\theta_{t}^{*}$ would be the value providing the best historical CBDT performance. For large $\kappa$ s the investor would hardly differentiate between utilities; thus the chosen $\theta_{t}^{*}$ may be far from the value of $\theta$ maximizing the historical EU. The Appendix provides more discussion on the similarity functions.

\subsection{The Role of Case-Based Reasoning in Portfolio Selection}

The purely EU-maximizing investor with a risk aversion $\gamma$ has no need to be case-based, because following CBDT implies departures from Savage rationality. He would likely follow some of the conventional portfolio rules. Nevertheless, some investors may be concerned about MU and would like to account for it. Their decision process for uncertainty modeling can be formalized by case-based intuition. Our CBDT methodology quantifies MU in portfolio selection.

The case-based portfolio investor determines his model belief degree $\theta_{t}^{*}$ by maximizing his objective function $Q\left(\theta_{t}\right)$ for the given risk aversion $\gamma$. The optimal $\theta_{t}^{*}$ is calculated for the case-based strategy, characterized by parameters $(\kappa, \lambda)$. Thus the case-based approach is not really an improvement in the portfolio selection procedure but a possible pattern of the investor's behavior in the presence of MU. The investigation of case-based decision patterns via perception of uncertainty in portfolio application is a core point of our study. 
Considering the patterns of case-based decisions, we aim to answer two questions. The first one is which patterns of case-based decision making are successful. This is of importance because investors with given $\gamma$ following successful strategies $(\kappa, \lambda)$ would stay in the market. On the contrary, the non successful CBDT investors would lose the money and abandon the market in the long run. This intuitively shows that the prevailing type of CBDT investors would potentially follow some successful $(\kappa, \lambda)$ strategies. The second question is on the interpretation of these successful case-based strategies. Empirical study in Section 4 provides answers to both questions.

\section{Empirical Study}

The empirical study has the following structure. First we introduce the conventional non-CBDT portfolio rules. Then we describe the data and methodological details of the CBDT approach in portfolio selection. Finally, we provide and discuss the empirical evidence.

\subsection{Conventional Asset Allocation Procedures}

We consider the economy with $k$ risky and one risk-free assets. Our investor chooses the portfolio weights $\mathbf{w}$ by maximizing the mean-variance objective function for the portfolio return $r_{p}$ :

$$
\max _{\mathbf{w}} E\left(r_{p}\right)-\frac{\gamma}{2} V\left(r_{p}\right) \quad \text { with } \quad r_{p}=\mathbf{w}^{\prime} \mathbf{r}+\left(1-\mathbf{w}^{\prime} \mathbf{1}\right) r_{f}
$$

where $\gamma$ denotes a risk aversion coefficient, $\mathbf{r}$ is a $k$-dimensional vector of risky asset returns with $E(\mathbf{r})=\boldsymbol{\mu}, V(\mathbf{r})=\boldsymbol{\Sigma}$. The problem (9) corresponds to the maximization of the expected quadratic utility. Now we introduce several approaches for selecting the portfolio weights w.

\subsubsection{Markowitz-Based Procedures}

The classical Markowitz optimal weights depend on the unknown true distribution parameters $(\boldsymbol{\mu}, \boldsymbol{\Sigma})$. We use the classical sample estimators $(\hat{\boldsymbol{\mu}}, \hat{\boldsymbol{\Sigma}})$ based on $n$ past returns. The true $\mathbf{w}$ and estimated $\hat{\mathbf{w}}$ Markowitz optimal weights are given by

$$
\mathbf{w}=\frac{1}{\gamma} \boldsymbol{\Sigma}^{-1}\left(\boldsymbol{\mu}-r_{f} \mathbf{1}\right), \quad \text { and } \quad \hat{\mathbf{w}}=\frac{1}{\gamma} \hat{\boldsymbol{\Sigma}}^{-1}\left(\hat{\boldsymbol{\mu}}-r_{f} \mathbf{1}\right) .
$$

The practical application of Markowitz weights (10) often leads to unsatisfactory results primarily due to estimation errors in $\hat{\boldsymbol{\mu}}$ and $\hat{\boldsymbol{\Sigma}}$ (cf. Michaud). Estimation risk could be mitigated by various methods, discussed in Section 2.1. For its reduction we combine the advantages of Jorion's Bayesian approach and the shrinkage methodology of Ledoit and Wolf. The shrunk covariance matrix is estimated according to

$$
\hat{\mathbf{\Sigma}}_{\mathrm{LW}}=\eta \hat{\mathbf{\Sigma}}+(1-\eta) \mathbf{F}
$$


where $\hat{\boldsymbol{\Sigma}}$ is a sample covariance matrix, $\mathbf{F}$ is a covariance matrix from the one-factor model, and $\eta$ is a shrinkage coefficient defined by Ledoit and Wolf. The shrunk mean returns are computed according to the Jorion procedure:

$$
\hat{\boldsymbol{\mu}}_{\mathrm{J}}=\left(1-\phi_{j}\right) \hat{\boldsymbol{\mu}}+\phi_{j} \mu^{G} \mathbf{1}
$$

where $\hat{\boldsymbol{\mu}}$ denotes the sample mean, $\mu^{G}$ is the mean of the global minimum variance portfolio, and $\phi_{j}$ is a shrinkage coefficient defined by Jorion. The estimated optimal weights $\hat{\mathbf{w}}_{\text {J, Lw }}$ are obtained by substituting the shrinkage parameters $\hat{\boldsymbol{\mu}}_{\mathrm{J}}$ and $\hat{\boldsymbol{\Sigma}}_{\text {LW }}$ into the EU maximization problem (9).

\subsubsection{Simple Rules for Portfolio Selection}

Simple investment rules are not justified by the EU approach but yield good results in practical applications. The recent overview of these methods is given in DeMiguel et al. Here we consider the two simple equal-weight approaches widely used in practice. The first alternative is to choose equal weights for all $k$ risky and the risk-free assets. The weights of risky assets and the fraction of wealth invested riskily are given by

$$
w_{i}=\frac{1}{k+1}, \quad \text { and } \quad \sum_{i=1}^{k} w_{i}=\frac{k}{k+1}
$$

The second alternative is based on the capital market line (CML) consideration and is developed in spirit of Black and Litterman. The investor chooses equal weights for all risky assets and constructs the composite asset with return $r_{c t}=\left(r_{1 t}+\ldots+r_{k t}\right) / k$. Then the portfolio problem is reduced to allocation between the composite and risk-free assets. We use the sample estimator for the composite asset variance $\hat{\sigma}_{c}^{2}$ and estimate the mean $\hat{\mu}_{c}$ along the CML by

$$
\hat{\mu}_{c}^{*}=r_{f}+\frac{\hat{\sigma}_{c}}{\sigma_{M}}\left(\mu_{M}-r_{f}\right)
$$

where $\left(\mu_{M}, \sigma_{M}^{2}\right)$ are the long-term parameters of the market portfolio proxy. Then the fraction of wealth invested in the composite risky asset is given by

$$
w_{c}=\frac{1}{\gamma} \frac{\hat{\mu}_{c}^{*}-r_{f}}{\hat{\sigma}_{c}^{2}}
$$

so the weight of each risky asset is given by $w_{i}=w_{c} / k$ for $i=1, \ldots, k$. This approach combines the advantages of both Markowitz and CAPM procedures.

\subsubsection{Constrained Markowitz-based Portfolio Selection}

Constraining portfolio weights improves Markowitz-based procedures. Introducing constraints is advocated among others by Frost and Savarino (1988), Grauer and Shen (2000), and Jagannathan and Ma (2003). It avoids extreme portfolio positions while preserving the advantages of Markowitz rules. These procedures provide a good out-of-sample portfolio performance in practical applications, 
see DeMiguel et al. Note that analytical solutions for constrained optimization problems are often unavailable; consequently numerical methods should be use. We implement the constraints by solving the problem (9) with the portfolio weights of risky assets restricted by

$$
0 \leq w_{i} \leq 1 / a \quad \text { for } \quad i=1, \ldots, k .
$$

We impose equal constraints for all risky assets. The choice $a=k$ guarantees $\mathbf{w}^{\prime} \mathbf{1} \leq 1$, implying no risk-free borrowing or lending.

\subsection{Data and Methodological Issues}

In our study the US investor allocates his wealth to risky assets listed in the Dow Jones Industrial Average Index (DJIA). The US 3-month T-Bill rate serves as a proxy for the risk-free rate. The asset returns are based on weekly prices for the period from 01.01.1973 to 06.10.2005, in total 1709 observations, for $k=24$ stocks permanently listed in the DJIA. The October 1987 crash has induced improvements in financial market regulation. In order to investigate whether our findings are valid for recent history, we report the results for $k=30$ Dow Jones stocks for the subperiod from 01.01.87 to 06.10.2005, in total 980 observations. All data is taken from DataStream.

The CBDT approach is applied to three groups of portfolio rules. The first group consists of pure EU-maximization approaches, namely simple Markowitz strategy (10), the Jorion approach (12) and the combined Jorion and Ledoit \& Wolf approach $(11,12)$. The equal weight approach (13) and equal weight strategy based on the CML consideration (15) constitute the second group. The annualized parameters of the market portfolio proxy are taken to be $\mu_{M}=0.13$ and $\sigma_{M}=0.21$ as in Farrell (1997, p.46). The third group consists of the adjusted EU-approaches, namely constrained procedures from the first group. We prohibit short selling and restrict the portfolio weights to $[0,1 / k]$ for all risky assets. The risk-free rate serves as a basic benchmark.

The expected utilities (9) are reported for all non-CBDT and CBDT approaches for the investor with given $\gamma=\{2,10,25\}$ and different case-based strategies $(\lambda, \kappa)$. The chosen values of the memory parameter $\lambda \in\{0,0.1,0.5,0.9,1.0\}$ correspond to perfect, good, average, poor and no memory, respectively. The indifference parameter of the similarity function takes the values $\kappa \in\{0.2,1,3.0\}$, defining convex, linear and concave functional forms. The moments in (9) are computed from the whole sample of realized portfolio returns. The non-CBDT portfolio weights are calculated as in Section 4.1. The respective CBDT portfolio weights are estimated by (2) with $\theta$ computed from (3) with the modulus similarity function. Other similarity functions do not alter the results significantly.

As in Ledoit et al. (2003), we estimate the parameters $\left(\boldsymbol{\mu}, \boldsymbol{\Sigma}, \sigma_{c}^{2}\right)$ with $n=104$ weekly returns. The choice of other estimation periods $(n=78,130)$ does not change the reported evidence. The history of portfolio returns is needed for estimating portfolio expectation and variance for constructing the CBDT objective function $Q\left(\theta_{t}\right)$. Thus we introduce a learning period for computing $E\left(r_{p}\right)$ and $V\left(r_{p}\right)$. The first case-based decision is conducted at the end of the learning period at $t=0$. The initial aspiration $H_{0}$ equals the average risk-free rate over the learning period. The one year (52 weekly observations) learning period is sufficient for getting reliable estimators of $E\left(r_{p}\right), V\left(r_{p}\right)$. Their precision is constantly improved by taking all newly incoming $r_{p}$ s for estimation purposes. The casebased investor with a given $\gamma$ chooses the optimal $\theta_{0}^{*}$ by maximizing $Q\left(\theta_{0}\right)$ for all $\kappa$ values. The next period aspiration $H_{1}$ is obtained according to (6) updating for all $\lambda \mathrm{s}$. 


\subsection{Empirical Results: Description}

We report the effect of introducing case-based reasoning on portfolio performance. The best CBDT strategies are identified and interpreted from an economic viewpoint. Finally, a graphical illustration highlights the intuition behind case-based decision making. Thee EUs for both non-CBDT and CBDT strategies based on the out-of-sample portfolio returns are presented in Table 1 for the period 1973-2005 (24 risky assets) and in Table 2 for the period 1987-2005 (30 risky assets) for given $\gamma \mathrm{s}$ and different constellations of $(\kappa, \lambda)$.

The non-CBDT investor with risk aversion $\gamma=2$ obtains the best overall results for the CML approach. The CBDT-CML approach is of interest for the investor with good memory $\lambda \gtrsim 0$ and the concave similarity function with indifference $\kappa>1$, which implies a high degree of similarity between different acts. However, the advantages of the CBDT-CML approach decrease in the period 1987-2005 compared to the whole sample 1973-2005. The non-CBDT investor with $\gamma=\{10,25\}$ gets the best results with the CML approach, too. However, the CBDT-adjusted Markowitz rules are superior. They provide the best performance for the convex similarity function with a large distance even between similar acts. The memory $\lambda \approx 0.5$ is of advantage for $\gamma=10$, while for $\gamma=25$ the best results are obtained for the weak memory $\lambda \approx 0.9$, implying quick aspiration adjustments. These findings are even more pronounced in the recent history of financial markets from 1987 to 2005. The purely Markowitz-based approaches underperform the risk-free one. For this class of strategies, the CBDT investor would avoid risky assets and invest almost exclusively in the risk-free one. This evidence holds for all $\gamma_{\mathrm{s}}$ and is robust with respect to the sub-sample selection.

Thus we identify two CBDT strategies with the best performance for the considered portfolio rules. The investor with small risk aversion should prefer the strategy with large indifference parameter $\kappa>$ 1 and nearly perfect memory $\lambda \gtrsim 0$. Such an investor hardly differentiates between acts with favorable and unfavorable outcomes and heavily relies on historical information. He can be characterized as a fundamental investor, following a variation of passive portfolio strategies. This approach implies gradual changes of model belief degree and the presence of anchoring and conservatism behavioral biases. Alternatively, the investor with large risk aversion should select a strategy with small $\kappa<1$ and large $\lambda \lesssim 1$. Then the model belief degree would quickly react to recent incoming information. Such an actively trading investor may exhibit representativeness and availability biases.

Figure 1 illustrates the time evolution of the model belief degree $\theta_{t}^{*}$ for the CML and constrained Markowitz-Jorion strategies. The CBDT parameters are $\kappa=3.0, \lambda=0.1$ for $\gamma=2$ and $\kappa=0.2, \lambda=$ 0.9 for $\gamma=10$. An increase in $\lambda$ and decrease in $\kappa$ accelerate the model belief adjustment. The time evolution of $\theta_{t}^{*}$ is characterized by three domains of attraction $\theta^{d}=\{0,0.5,1\}$. They denote the states of complete disbelief, average and complete belief. The optimal $\theta_{t}^{*}$ strongly depends on the past performance of the underlying non-CBDT strategies and of the risk-free rate evolution. The optimal $\theta_{t}^{*}$ from the interval $[0.5,1]$, characterizing strong belief degree, can hardly switch to the interval $[0,0.5]$, characterizing weak belief, and vice versa. Previous investment failures increase the disappointment of investor. On the contrary, past successes increase the investor's confidence, stimulating him to invest more in risky assets. The technical motivation for this effect is provided in the Appendix. The aspiration level on Figure 2 visualizes the evolution of the investor's satisfaction. It varies around the risk-free rate and exhibits clusters coinciding with the periods of low and high market volatility. This agrees with March and Simon's proposition: "Over time, the aspiration level 
tends to adjust to the level of achievement ... the level of satisfactory performance is likely to be very close to the actually achieved level of recent performance" (pp. 182-183).

\subsection{Empirical Results: Discussion}

The CBDT investor allows departures from rationality. Two successful case-based patterns premise completely different behavior. The fundamental trading approach is based on perfect memory, with low differentiation between acts. On the contrary, active trading exploits recent information and profits from high differentiation between favorable and unfavorable outcomes. Consequently, fundamental and active traders exhibit different behavioral biases. Now we provide an economic explanation for why these case-based strategies are of interest.

Fundamental CBDT trading implies making decisions relying on good memory about acts and outcomes. This would bring the highest benefit in situations with predictable returns and/or their second moments. Predictability on asset markets points to departures from efficiency. Thus the fundamental strategy may be more successful in markets, which are rather far from efficiency due to limits to arbitrage. In our empirical study we investigate the success of CBDT on the established US market. We consider the entire sample from 1973 to 2005 as well as two subsamples, from 1973 to 1986 and from 1987 to 2005. The predictability of the utility function could be separated into predictability of portfolio return and predictability of portfolio variance. We illustrate the predictability by providing the autocorrelations for the first and second moments of the equal weight portfolio return for all periods, considered in the study.

Table 3 reports a drastic decrease of autocorrelations in the subperiod 1987-2005 compared to whole period 1973-1986. This suggests that the US market updates more precisely upon new information. This indicates a decrease of predictability and increase of efficiency. Comparing the case-based results for the period 1973-2005 with the results for the period 1987-2005 confirms the evidence that the US market is becoming more efficient. Benefits from the fundamental strategy have reduced over time. This can be interpreted as support for the hypothesis that acceleration of information transfer improves arbitrage in the market. From this viewpoint the fundamental CBDT investors would abandon efficient markets in the long run. On the contrary, the benefits from case-based active trading have increased in the period 1987-2005. Active trading exploits the latest information and can be interpreted as arbitrage. Thus the active CBDT strategy serves to improve market efficiency. Consequently, such traders would survive in the long run.

This empirical evidence supports the CBDT application for the US market investor. The best identified case-based strategies may mimic widely observed behavioral biases. The success of fundamental and active trading CBDT strategies is linked to the degree of market efficiency. This stresses the importance of case-based decision making for general uncertainty assessment.

\section{Summary}

In this paper we quantify general uncertainty in portfolio selection using the case-based reasoning of Gilboa and Schmeidler (2001a). The situations with general uncertainty are characterized by an absence of information both about possible states of nature and their probabilities. Case-based 
Figure 1: The dynamics of the optimal degree of model belief $\theta_{t}^{* a}$ CML strategy with $\gamma=2$

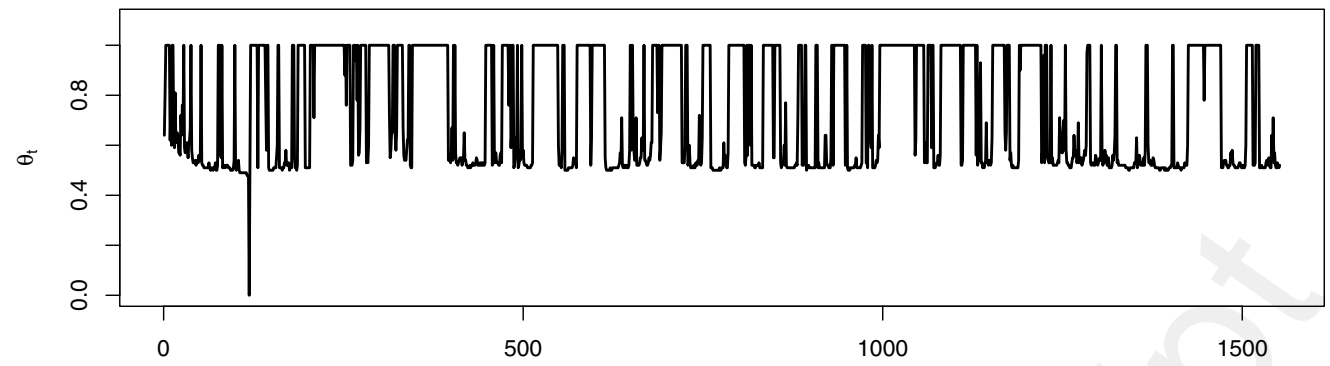

CML strategy with $\gamma=25$

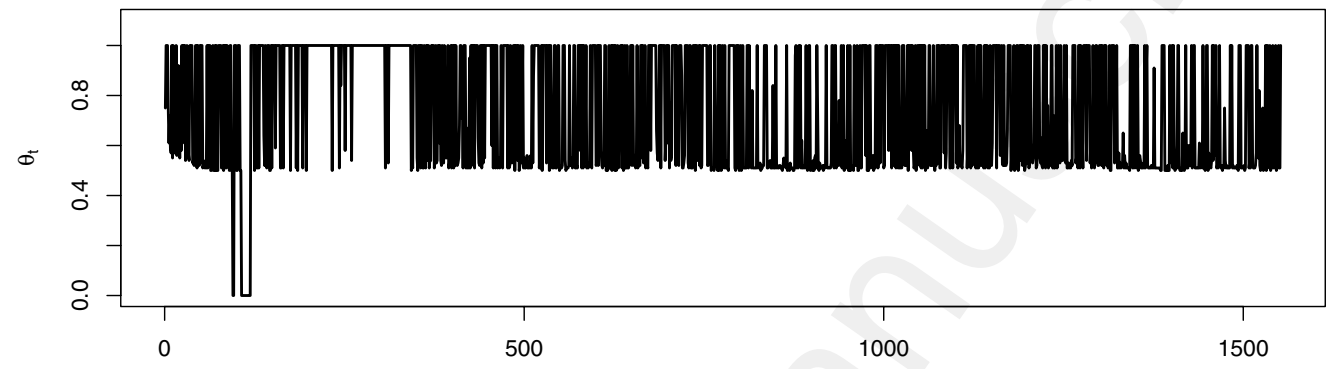

Constrained Markowitz-Jorion strategy with $\gamma=2$

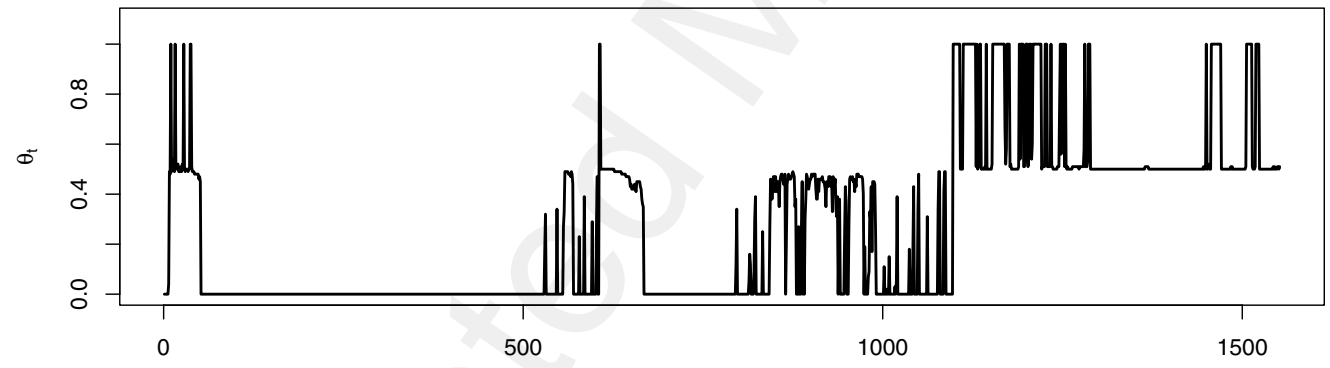

Constrained Markowitz-Jorion strategy with $\gamma=25$

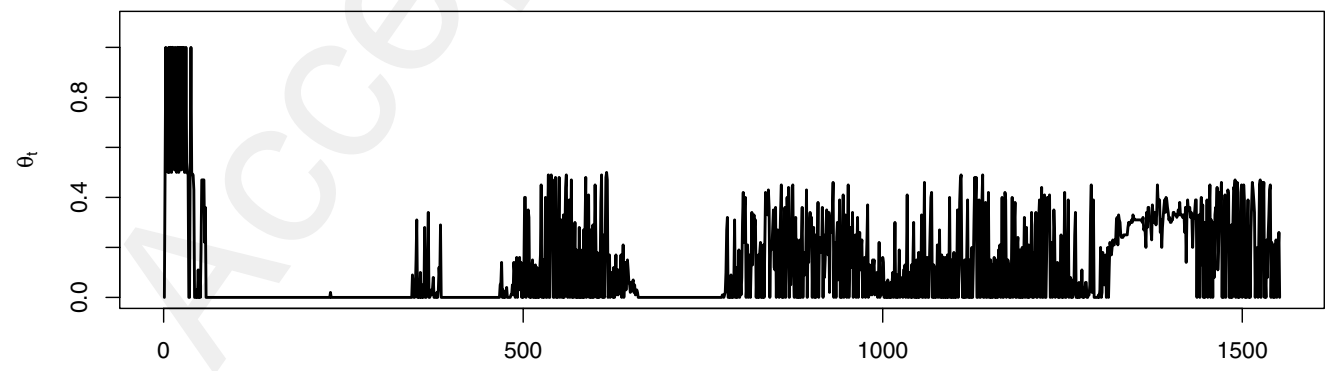

${ }^{a}$ The indifference parameter $\kappa$ is set to 3 and the memory parameter $\lambda$ to 0.1 for the strategies with $\gamma=2$ and to 0.2 and to 0.9 for the strategies with $\gamma=25$, respectively. 
Figure 2: The dynamics of the aspiration level $H_{t}$ and risk-free rate $r_{f}{ }^{a}$

CML strategy with $\gamma=2$

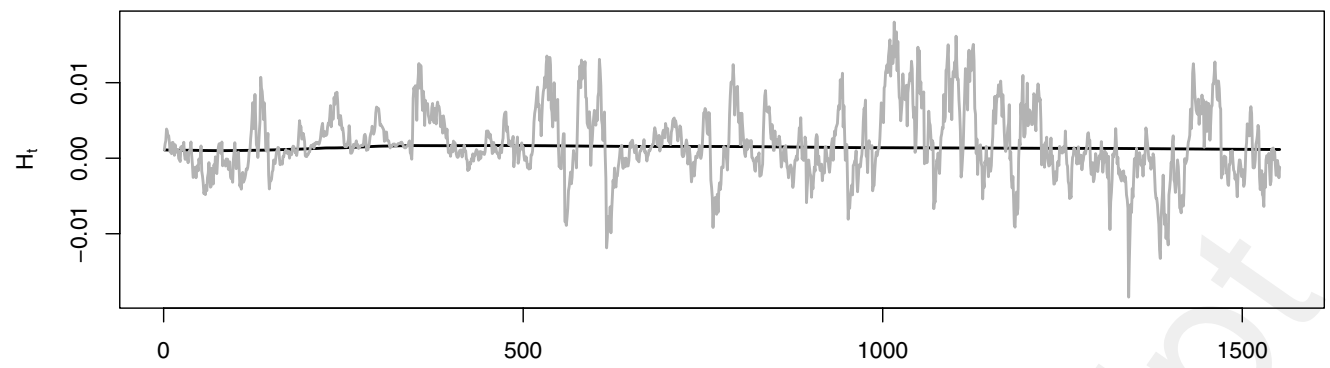

CML strategy with $\gamma=25$

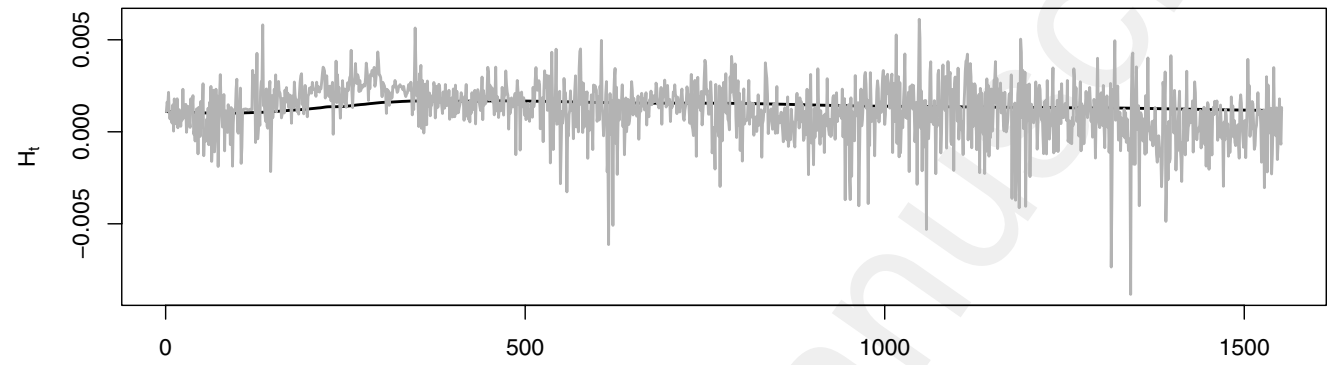

Constrained Markowitz-Jorion strategy with $\gamma=2$

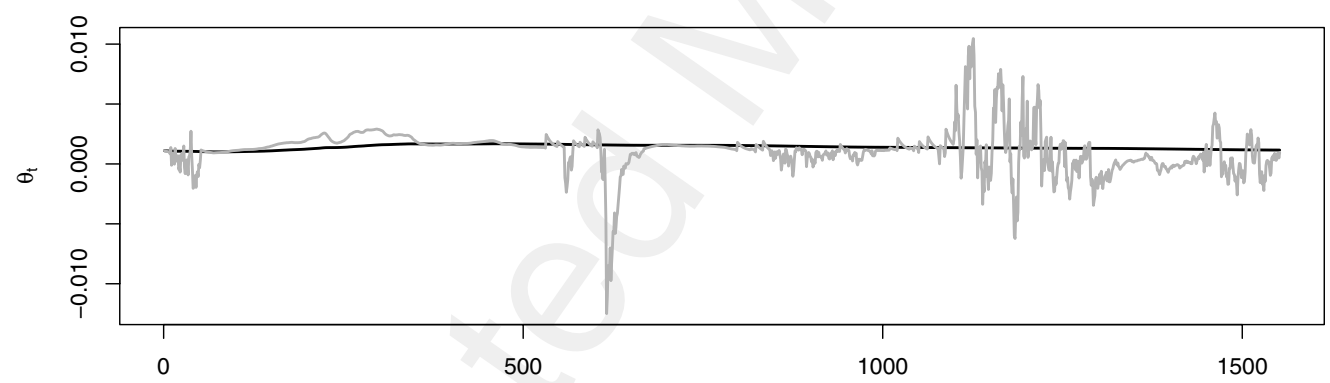

Constrained Markowitz-Jorion strategy with $\gamma=25$

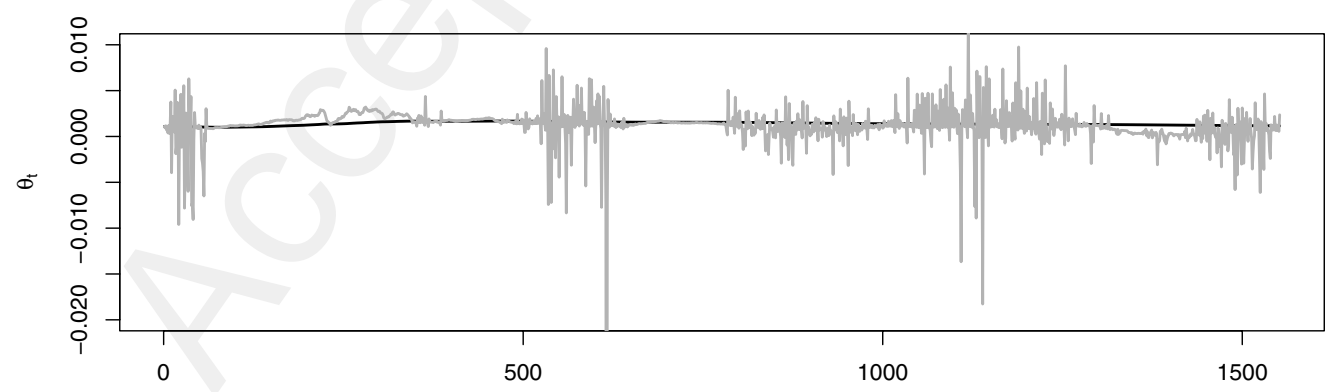

${ }^{a}$ The indifference parameter $\kappa$ is set to 3 and the memory parameter $\lambda$ to 0.1 for the strategies with $\gamma=2$ and to 0.2 and to 0.9 for the strategies with $\gamma=25$, respectively. 
Table 1: Expected utilities with and without CBDT reasoning for the period $1973-2005^{a}$

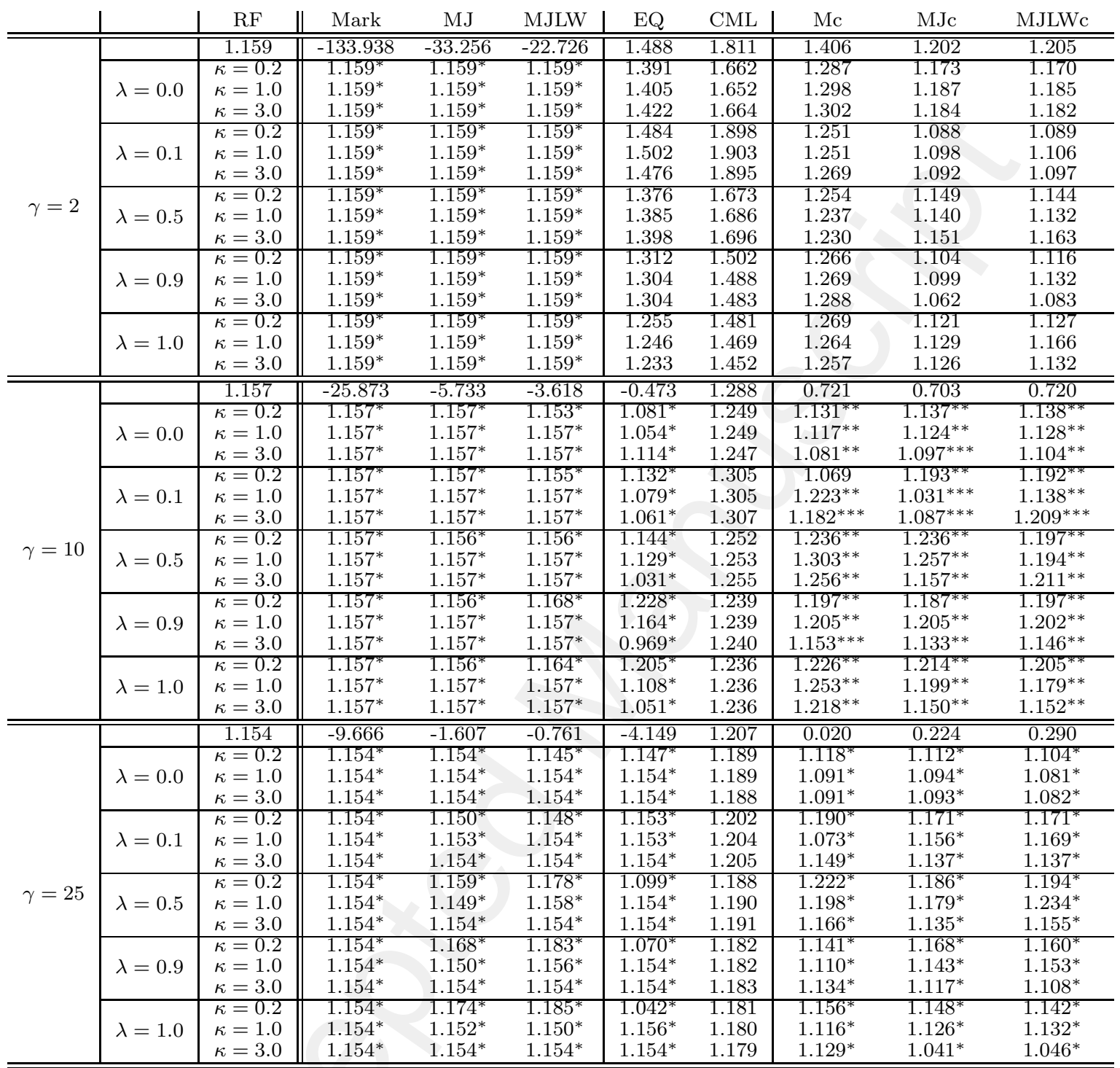

${ }^{a}$ For each value of $\gamma$ the first line contains the non-CBDT expected utilities; the rest of the block contains the CBDTutilities with the given values of memory $\lambda$ and indifference parameters $\kappa$. Mark denotes the classical Markowitz approach, $M J$ the Markowitz approach with the mean estimated as in Jorion, $M J L W$ the Markowitz approach with the mean estimated as in Jorion and the covariance matrix as in Ledoit and Wolf, $E Q$ the strategy with equal portfolio weights, $C M L$ the approach based on capital market line considerations, $M c$ constrained Markowitz approach, $M J c$ constrained $M J$ strategy, and $M J L W c$ constrained $M J L W$ strategy. The CBDT strategies with significantly higher EUs than the non-CBDT strategy are marked with ${ }^{*}$ for $1 \%$ significance level, ${ }^{* *}$ for $5 \%$ and *** for $10 \%$. The number of assets in the portfolio is equal to 24 . 
Table 2: Expected utilities with and without CBDT reasoning for the period 1987-2005

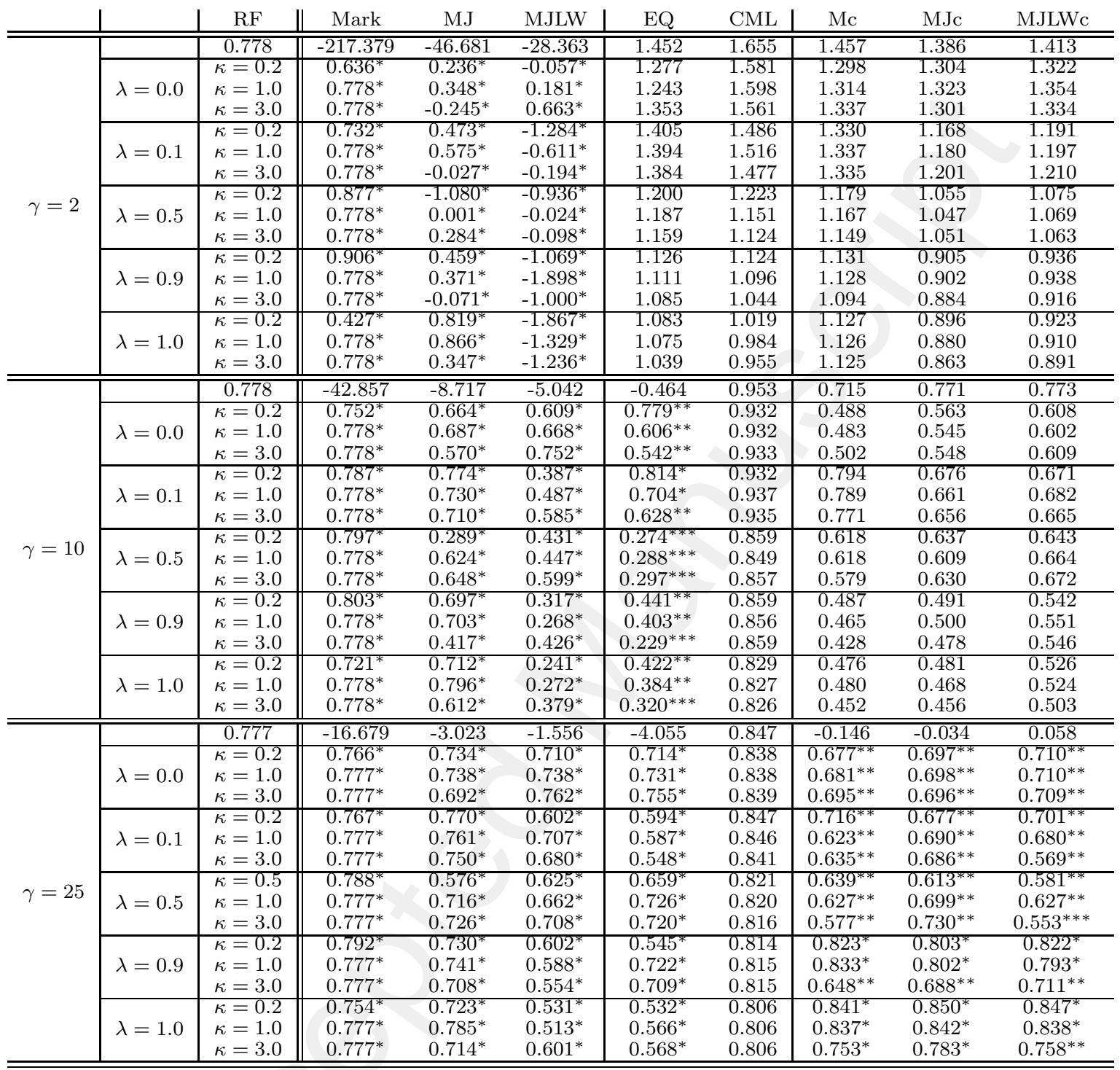

${ }^{a}$ For each value of $\gamma$ the first line contains the non-CBDT expected utilities; the rest of the block contains the CBDTutilities with the given values of memory $\lambda$ and indifference $\kappa$ parameters. Mark denotes the classical Markowitz approach, $M J$ the Markowitz approach with the mean estimated as in Jorion, $M J L W$ the Markowitz approach with the mean estimated as in Jorion and the covariance matrix as in Ledoit and Wolf, $E Q$ the strategy with equal portfolio weights, $C M L$ the approach based on capital market line considerations, $M c$ constrained Markowitz approach, $M J c$ constrained $M J$ strategy, and $M J L W c$ constrained $M J L W$ strategy. The CBDT strategies with significantly higher EUs than the non-CBDT strategy are marked with ${ }^{*}$ for $1 \%$ significance level, ${ }^{* *}$ for $5 \%$ and *** for $10 \%$. The number of assets in the portfolio is equal to 30 . 
Table 3: Autocorrelations of the equal weight portfolio returns and squared returns for different investment periods.

\begin{tabular}{c|c||cccccc} 
& period & 1 & 2 & 3 & 4 & 5 & 6 \\
\hline \hline \multirow{3}{*}{$\mathrm{ACF}$ of $r_{p}$} & $1973-1986$ & -0.0342 & 0.0018 & $0.1065^{*}$ & -0.0541 & -0.0358 & 0.0220 \\
& $1987-2005$ & -0.0204 & 0.0230 & 0.0221 & -0.0258 & -0.0405 & $0.0731^{* *}$ \\
& $1973-2005$ & -0.0250 & 0.0154 & $0.0525^{* *}$ & -0.0351 & -0.0367 & $0.0566^{* *}$ \\
\hline \multirow{3}{*}{$\mathrm{ACF}$ of $r_{p}^{2}$} & $1973-1986$ & $0.2865^{*}$ & $0.1832^{*}$ & $0.2156^{*}$ & $0.2056^{*}$ & $0.1643^{*}$ & $0.1982^{*}$ \\
& $1987-2005$ & $0.0878^{*}$ & $0.0667^{* *}$ & 0.0390 & 0.0515 & 0.0260 & $0.1650^{*}$ \\
& $1973-2005$ & $0.1156^{*}$ & $0.0835^{*}$ & $0.0637^{*}$ & $0.0736^{*}$ & $0.0462^{* * *}$ & $0.1706^{*}$
\end{tabular}

decision theory (CBDT) amends the EU approach for such situations. We define general uncertainty as the degree of the investor's belief in the possibility of benefit from conventional investment rules. The CBDT approach is suggested for selecting the degree of model belief. The major aim of the study is to identify and interpret successful patterns of case-based decisions.

The case-based investor determines his model belief degree to maximize the objective function, defined as the sum of excess utilities for all hypothetical acts weighted by similarity of these acts to the act of choice. Hypothetical reasoning is appropriate because the investor can easily construct the consequences of all possible past choices. The indifference parameter determines the shape of the similarity function and serves to measure the distance between acts. The excess utility is calculated as the difference of the hypothetical utility and aspiration level, serving as a level of investor satisfaction. The memory strength determines the speed of aspiration adjustment. Indifference and memory parameters formalize the case-based strategy for a given investor's risk aversion. The model belief degree is used for linear adjustment of conventional portfolio rules.

The proposed case-based methodology is investigated in the empirical study. Based on our findings, we identify two successful case-based strategies. The case-based investor with a small risk aversion should choose a strategy implying gradual adjustments of model belief degree. This strategy provides the best results in a non-efficient market with high predictability degree. On the contrary, the case-based investor with medium and large risk aversion benefits from a strategy with quick adjustments. This implies active trading with immediate reaction on newly incoming information and market efficiency improvement. Thus the successful case-based approaches provide motivation for various investment strategies. They incorporate some behavioral phenomena observed on financial markets and could be seen as a type of decision-making rules under bounded rationality.

\section{Acknowledgements}

We would like to express our gratitude to two anonymous referees for valuable comments. Special thanks are addressed to Thomas Lux for the helpful discussion of the paper. Any remaining errors are of our own responsibility. 


\section{References}

Aiolfi, M., Favero, C., 2005. Model uncertainty, thick modeling and the predictability of stock returns. Journal of Forecasting 24, 233-254.

Avramov, D., 2002. Stock predictability and model uncertainty. Journal of Financial Economics 64, 423-458.

Barberis, N., Thaler, R., 2003. A survey of behavioral finance. In: Constantinides, G.M., Harris, M., Stulz, R.M. (Eds.). Handbook of the Economics of Finance, Vol. I. Amsterdam: Elsevier, 1053-1128.

Best, M., Grauer, R., 1991. On the sensitivity of mean-variance-efficient portfolios to changes in asset means. Review of Financial Studies 4, 315-342.

Billot, A., Gilboa, I., Samet, D., Schmeidler, D., 2005. Probabilities as similarity-weighted frequencies. Econometrica 73, 1125-1136.

Black, F., Litterman, R., 1992. Global portfolio optimization. Financial Analyst Journal 48/5, 28-43.

Blonski, M., 1999. Social learning with case-based decisions. Journal of Economic Behavior and Organization 38, 59-77.

Boergers, T., Sarin, R., 2000. Naive reinforcement learning with endogenous aspirations. International Economic Review 41, 921-950.

Chamberlain, G., 2000. Econometric applications of maximin expected utility. Journal of Applied Econometrics 15, 625-644.

DeMiguel, A-V., Garlappi, L., Uppal, R., 2008. Optimal versus naive diversification: how inefficient is $1 / N$ portfolio strategy. Review of Financial Studies, in press.

Dequech, D., 2006. The new institutional economics and the theory of behaviour under uncertainty. Journal of Economic Behaviour and Organization 59, 109-131.

Farrell, J.L., 1997. Portfolio Management: Theory and Applications. New York: McGraw-Hill.

Frost, P.A., Savarino, J.E., 1988. For better performance: constrain portfolio weights. Journal of Portfolio Management 15/1, 29-34.

Garlappi, L., Uppal, R., Wang, T., 2007. Portfolio selection with parameter and model uncertainty. Review of Financial Studies 20, 41-81.

Gilboa, I., Schmeidler, D., 1989. Maxmin expected utility with non-unique prior. Journal of Mathematical Economics 18, 141-153.

Gilboa, I., Schmeidler, D., 1995. Case-based decision theory. Quarterly Journal of Economics 110, 605-639.

Gilboa, I., Schmeidler, D., 1997a. Act similarity in case-based decision theory. Economic Theory 9, 47-61. 
Gilboa, I., Schmeidler, D., 1997b. Cumulative utility consumer theory. International Economic Review $38,737-761$.

Gilboa, I., Schmeidler, D., 2001a. A Theory of Cased-Based Decisions. Cambridge, UK: Cambridge University Press.

Gilboa, I., Schmeidler, D., 2001b. A cognitive model of individual well-being. Social Choice Welfare $18,269-288$.

Gilboa, I., Schmeidler, D., Wakker, P.P., 2002. Utility in case-based decision theory. Journal of Economic Theory 105, 483-502.

Golosnoy, V., Okhrin, Y., 2007. Multivariate shrinkage for optimal portfolio weights. European Journal of Finance 13, 441-458.

Grauer, R., Shen, F., 2000. Do constraints improve portfolio performance? Journal of Banking and Finance 24, 1253-1274.

Hanaki, N., Sethi, R., Erev, I., Peterhansl, A., 2005. Learning strategies. Journal of Economic Behavior and Organization 56, 523-542.

Hansen, L.P., Sargent, T.J., 2001. Robust control and model uncertainty. American Economic Review $92,60-66$.

Jagannathan, R., Ma, T., 2003. Risk reduction in large portfolios: why imposing the wrong constraints helps. Journal of Finance 58, 1651-1683.

Jahnke, H., Chwolka, A., Simons, D., 2005. Coordinating service-sensitive demand and capacity by adaptive decision making: an application of case-based decision theory. Decision Sciences 36, $1-32$.

Jorion, P., 1986, Bayes-Stein estimation for portfolio analysis. Journal of Financial and Quantitative Analysis 21, 293-305.

Kahneman, D., Tversky, A., 1979. Prospect theory: an analysis of decision under risk. Econometrica 47, 263-291.

Kan, R., Zhou, G., 2006. Optimal estimation for economics gains: portfolio choice with parameter uncertainty. Journal of Financial and Quantitative Analysis, in press.

Klein, R., Bawa, V., 1976. The effect of estimation risk on optimal portfolio choice. Journal of Financial Economics 3, 215-231.

Knight, F.H., 1921. Risk, Uncertainty and Profit. Boston: Houghton Mifflin.

Kogan, L., Wang, T., 2003. A simple theory of asset pricing under model uncertainty. Working paper, available at http://web.mit.edu/lkogan2/www/KoganWang2002.pdf

Kumar, P., 2006. Learning about investment risk: The effect of structural uncertainty on dynamic investment and consumption. Journal of Economic Behavior and Organization 60, 205-229. 
Ledoit, O., Santa-Clara, P., Wolf. M., 2003. Flexible multivariate GARCH modeling with an application to international stock markets. Review of Economics and Statistics 85, 735-747.

Ledoit, O., Wolf, M., 2003. Improved estimation of the covariance matrix of stock returns with an application to portfolio selection. Journal of Empirical Finance 10, 603-621.

MacLeod, W. B., Pingle, M., 2005. Aspiration uncertainty: its impact on decision performance and process. Journal of Economic Behavior and Organization 56, 617-629.

March, J., Simon, H., 1958. Organizations. New York: John Wiley.

Markowitz, H., 1952. Portfolio selection. Journal of Finance 7, 77-91.

Matsui, A., 2000. Expected utility and case-based reasoning. Mathematical Social Sciences 39, 1-12.

Michaud, R.O., 1998. Efficient Asset Management. Boston: Harvard Business School Press.

Oechssler, J., 2002. Cooperation as a result of learning with aspiration levels. Journal of Economic Behavior and Organization 49, 405-409.

Pastor, L., 2000. Portfolio selection and asset pricing models, Journal of Finance 55, 179-223.

Pastor, L., Stambaugh, R., 2000. Comparing asset pricing models: an investment perspective. Journal of Financial Economics 56, 335-381.

Polson, N., Tew, B., 2000. Bayesian portfolio selection: an empirical analysis of the S\&P index 1970-1996. Journal of Business and Economic Statistics 18, 164-173.

ter Horst, J., de Roon, F., Werker, B., 2006. Incorporating estimation risk in optimal portfolios. In: Renneboog, L.D.R. (Ed.). Advances in Corporate Finance and Asset Pricing, Amsterdam: Elsevier, 449-472.

Tu, J., Zhou, G., 2004. Data-generating process uncertainty: what difference does it make in portfolio decisions? Journal of Financial Economics 72, 385-421.

Wang, Z., 2005. A shrinkage approach to model uncertainty and asset allocation. Review of Financial Studies 18, 673-705. 


\title{
General Uncertainty in Portfolio Selection: A Case-Based Decision Approach
}

\author{
Vasyl Golosnoy ${ }^{a, *}$ and Yarema Okhrin ${ }^{b}$ \\ ${ }^{a}$ Institute of Statistics and Econometrics, University of Kiel, Germany \\ * Corresponding author. E-mail address: vgolosnoy@stat-econ.uni-kiel.de, \\ Phone: +49 (0) 4318804381 , Fax: +49 (0) 4318807605 \\ ${ }^{b}$ Department of Statistics, European University, Frankfurt (Oder), Germany \\ E-mail address: okhrin@euv-frankfurt-o.de
}

\begin{abstract}
Often a portfolio investor can hardly imagine all states of nature relevant to his investment problem, causing general uncertainty concerning an asset allocation model. We quantify general uncertainty as the weakness of an investor's belief in a conventional portfolio procedure, then we develop the case-based decision making approach for determining the optimal belief degree. The economic effect of the proposed case-based methodology is investigated in the empirical study. The empirical results suggest two successful patterns of case-based decisions that could be linked to the issue of market efficiency. Moreover, our case-based modeling reflects some behavioral phenomena observed on financial markets.
\end{abstract}

Keywords: act similarity, case-based reasoning, general uncertainty, model belief degree. JEL classification: D81, G11, G14 